\title{
Evaluation of POSSUM and P-POSSUM as predictors of mortality and morbidity in patients undergoing laparotomy at a referral hospital in Nairobi, Kenya
}

\author{
Kimani MM ${ }^{1,2 *}$, Kiiru JN ${ }^{3}$, Matu $\mathrm{MM}^{3}$, Chokwe $\mathrm{T}^{1,2}$, Saidi $\mathrm{H}^{1,2}$ \\ Institutional affiliations: 1. University of Nairobi, College of Health Sciences, 2. Kenyatta National Hospital, Department of surgery, \\ 3. Kenya Medical Research Institute, Centre for Microbiology Research *Corresponding Author: Moses Kimani, P.0. Box 54270- \\ 00200 Nairobi Kenya, Tel: +254724400193, Email: mwangikimanimoses@gmail.com
}

\begin{abstract}
\section{Background}

The Physiological and Operative Severity Score for enUmeration of Morbidity and Mortality (POSSUM) and its Portsmouth modification (P-POSSUM) were developed for comparative audit in surgical patients. This study evaluated applicability of these systems in estimating mortality and morbidity risks in a cohort of patients undergoing laparotomy at the national referral hospital in Nairobi, Kenya. Methods

Data of 166 patients undergoing laparotomy was subjected to POSSUM and P-POSSUM scoring systems and analyzed using linear and exponential methods. The discrimination power of POSSUM and P-POSSUM as predictors of surgical outcome was measured using the receiver-operating characteristic (ROC) curve.
\end{abstract}

\section{Results}

The overall observed to expected (0:E) ratio using linear analysis was 0.29:1 (POSSUM) and 0.67:1 (P-POSSUM) while exponential analysis gave an 0:E of 0.2:1 (POSSUM) and 0.4:1 (P-POSSUM). The predicted morbidity using POSSUM was 1.09:1 (linear analysis) and 1:1 (exponential analysis). Hosmer-Lemeshow analysis did not show a significant lack of fit with the observed mortality when applied to POSSUM and P-POSSUM. The area under ROC curve was 0.74 (POSSUM) and 0.78 (P-POSSUM).

Conclusion

Our results support the suitability of P-POSSUM and POSSUM scoring systems to stratify morbidity and mortality risk in our study population.

\section{Introduction}

Surgical audit has become an essential requirement in modern surgical practice for ensuring the quality of care(1). By evaluating the procedures used for diagnosis and treatment and to lower the risks of patients undergoing surgery, both individual surgeon and institutional outcome trends can be analyzed (1).

Audits are common quality indicator tools in hospitals in the developed countries but rarely done in many developing countries (2). Patients are therefore subjected to surgery without due consideration of factors that may complicate the outcomes of these operations. In such setups, crude morbidity and mortality rates are used as indicators of quality of care. However, these approaches may be misleading since lack of adjustments for different settings leads to erroneous results when outcomes of surgery are compared between different units and hospitals $(3,4)$. In order to standardize surgical audit procedures, a number of scoring systems have been developed for the comparison of case mixes.
The Physiological and Operative Severity Score for the enUmeration of Mortality and Morbidity (POSSUM) (5) and its Portsmouth predictor modification (P-POSSUM) (6) audit systems use the patients' physiological and operative data to predict morbidity and mortality and compare surgical outcomes independent of case mix $(5,6)$. The two systems are built on the accepted principle that the mode of presentation, physiological condition of the patient and extent of the surgical procedure performed are predictors of patient outcome. This can be useful in monitoring the improvement of surgical outcomes in patients ranked to be at high risk of mortality and morbidity following surgery.

Our local experience with outcome predictions in any surgical field using standardized schemes is limited. Neither POSSUM nor P-POSSUM have been applied in any Kenyan hospitals and therefore, little is known about their applicability as indicators of quality of healthcare delivery to patients undergoing surgical procedures in Kenya. This study aimed to evaluate the applicability 
of POSSUM and P-POSSUM scoring systems in predicting post-surgical outcomes in patients undergoing laparotomy in a referral hospital in Nairobi, Kenya.

\section{Materials and methods}

Kenyatta National Hospital (KNH) is the central referral and teaching hospital in Kenya. It is located in the city of Nairobi. Nairobi has an estimated population of about 3 million people. Patients undergoing laparotomy at the hospital are managed by a tier of doctors from interns, senior house officers, surgical residents, registrars to full time consultants in General Surgery. However, all surgeries are performed by the residents, registrars and consultants.

The institution has the capacity to undertake major surgical procedures on a 24-hour basis. We conducted a single centre prospective study over a period of 6 months in patients undergoing laparotomy from the emergency unit and those admitted to the general surgical wards, intensive and high dependency units at $\mathrm{KNH}$. A total of 166 patients, undergoing either elective or emergency surgery, and meeting the recruitment criteria, were enrolled in the study.

Selection of the patients was done from the point of admission - emergency laparotomy patients were selected from the accident and emergency department while those who underwent elective laparotomy were recruited from different hospital wards. Patient data was collected from hospital files. Patients and their relatives provided further information during the consent process. The primary outcomes were the actual 30-day mortality rates for the overall group and for different risk strata $(6,7)$. Actual (observed) deaths were then compared to predicted (expected) mortality generated using the POSSUM and P-POSSUM equations. The results were analyzed using linear and exponential methods as described by Hobson et al (8). Secondary outcomes included actual and predicted rates of morbidities (surgical site infection, electrolyte imbalance, burst abdomen, intestinal ob- struction, and fecal fistulas among others) for the same group of patients, as originally described by Copeland et al (5). Pre-operative scores were done as described by Copeland et al (5) using 12 physiological factors while 6 operative scores were made at the end of the operation. Predicted morbidity and mortality rates were calculated using both POSSUM and P-POSSUM equations as described by Wijesinghe et al (9).

The discrimination power and accuracy of POSSUM and P-POSSUM in predicting death was measured using the receiver-operating characteristic (ROC) curve. An area under the ROC curve of less than 0.7 show poor discrimination, that of between 0.7 and 0.8 indicates a reasonable discriminatory power while a value above 0.8 suggests perfect discrimination. Statistical analyses and ROC curve plotting were done using the SPSS Version 15.0 (SPSS, Chicago, IL, USA). The Hosmer-Lemeshow (H-L) goodness of fit test (10) was used to assess the differences between predicted and observed mortality rates for POSSUM and P-POSSUM. A value of $\mathrm{p} \leq 0.05$ was considered statistically significant. 
Evaluation of POSSUM and P-POSSUM as predictors of mortality and morbidity in patients undergoing laparotomy at a referral hospital in Nairobi, Kenya Kimani $\mathrm{MM}^{1,2}$, Kiiru $\mathrm{JN}^{3}$, Matu $\mathrm{MM}^{3}$, Chokwe $\mathrm{T}^{1,2}$, Saidi $\mathrm{H}^{1,2}$

\begin{tabular}{llllll} 
Mortality & Number & Mean risk (\%) & Predicted & Actual deaths & 0:E ratio \\
\hline group (\%) & of patients & & (expected) deaths & (observed) (\%) & \\
\hline$<10$ & 135 & 2.5 & 3 & $4(3)$ & $1.2: 1$ \\
\hline $10-19$ & 14 & 14.8 & 2 & $1(7.1)$ & $0.5: 1$ \\
\hline $20-29$ & 6 & 24 & 1 & $1(16.7)$ & $0.7: 1$ \\
\hline $30-39$ & 5 & 38.6 & 2 & $1(20)$ & $0.5: 1$ \\
\hline $40-100$ & 6 & 54.5 & 3 & $1(16.7)$ & $0.5: 1$ \\
\hline $0-100$ & 166 & & 12 & 8 & $0.67: 1$ \\
\hline
\end{tabular}

to POsSum ( $\mathrm{p}=0.22)$ and P-POSSUM $(\mathrm{p}=0.58)$. The area under ROC curve for POSSUM was 0.74 and 0.78 for P-POSSUM. The ROC curve analysis showed reasonable discriminatory capability of mortality for both P-POSSUM and POSSUM scores.

\section{Discussion}

Table 3: P-POSSUM scores comparison of observed and predicted mortality using linear analysis

The results of the present study using POSSUM and P-POSSUM as predictive tools in consecutive patients undergoing abdominal surgery at Kenyatta National Hospital have allowed a reasonable assessment of the "quality of laparotomy" at the institution. The actual mortality rate of $4.8 \%$ recorded in the study is comparable to post-laparotomy mortality rates in earlier studies. Yii and $\mathrm{Ng}(2)$ in Malaysia recorded a mortality rate of $6.1 \%$ while Bollschweiler et al (12) found a death rate of $5.8 \%$ in their post-gastrectomy cohort.

\section{Results}

Of the 166 patients enrolled, 111 (67\%) were males. The ages ranged from 13 to 82 years (mean 35 years). Majority (67\%) were enrolled for emergency laparotomy. Eight $(4.8 \%)$ patients died. The overall predicted mortality of the study group was calculated at $16.7 \%$ using the POSSUM linear analysis method (table 1) and 50\% using POSSUM exponential analysis (table 2). This gave an observed to expected (O:E) ratio of 0.29:1 and 0.2:1 respectively. When similar calculations were made using the P-POSSUM scheme, the predicted mortality was 7.2\% (linear analysis) and 13.9\% (exponential analysis) giving an Observed to Expected ratio of 0.67:1 and 0.4:1 as shown in tables 3 and 4 respectively.

Eighty seven patients (52\%) developed post-operative complications. The overall predicted morbidity using POSSUM was 38\% (linear analysis) and 62\% (exponential analysis). These gave an observed to expected (O:E) morbidity ratio of 1.09:1 (linear analysis) and a ratio of 1:1 (exponential analysis) as shown in table 5 and table 6 respectively.

Hosmer-Lemeshow analysis did not show a significant lack of fit with the observed mortality when applied
The studies by Vather et al (13) and Khan et al. (14) report even lower rates of around $4 \%$.

The POSSUM mortality score over-predicts mortality in this study by a factor of 3 using exponential analysis and by a factor of 5 using linear analysis. It overestimated mortality in the low risk group by a factor of 2-3 and by factor of 3-5 in the high-risk group. Wijesinghe et al. (9) advocated for the use of exponential analysis in estimating risk using the POSSUM equation. However the "exponential" method of analysis and subgroup analysis were not clearly described in the original Possum publication (5). It was only later that Wijesinghe et al elaborated on the methods of analysis for POSSUM and P-POSSUM and the divisions used to categorise patients to high risk and low risk groups (9). In our study there was significant over-prediction of mortality, irrespective of the method of analysis used for POSSUM mortality.

In comparison, the P-POSSUM equation performed better in prediction with linear analysis. The overall $\mathrm{O}$ : E ratio was $0.67 \%$. This produced an overall over prediction factor of only 1.5 . It is important to note that P-POSSUM under predicated deaths in patients at low risk mortality group (<10\%) O:E 1.2:1. This under prediction in the low risk group has also been observed by 
other groups $(2,6,9,12)$. Majority of our patients fell within the low risk category (risk of mortality of less than 10\%). This is significant as patients with a very low risk of mortality are unlikely to have POSSUM or P-POSSUM assessment prior to surgery outside of an audit or research setting. PPOSSUM under-estimated mortality by a factor of two in high-risk patients, with a predicted mortality rate of over $40 \%$.

The overall morbidity rate of $52 \%$ in our study was in keeping with the rates observed in other parts of the world of between $25-65 \%(11,16)$.

POSSUM by exponential analysis, accurately predicted the overall morbidity in this study group (O:E ratio 1:1). When POSSUM morbidity equation was analysed using the linear method, it was found to have significant over prediction (factor of five) in the low risk group (5.48:1). This is in keeping with the analysis by Wejisinghe et al (9) and Hobson et al. (8) who advocated for its use, with exponential analysis only.

Using the ROC curve, both POSSUM and P-POSSUM mortality schemes recorded an area under the curve of between 0.7 and 0.8 meaning that there was a reasonable discriminatory power using both models with $\mathrm{P}$ POSSUM showing better prediction accuracy.

\section{Conclusion}

P-POSSUM (linear analysis) is a reliable predictor of post operative mortality, while POSSUM (exponential analysis) is a good predictor of post operative morbidity in our situation. POSSUM tends to over predict mortality and may need further reviews to make it more accurate.

\section{References}

1. Simon D Bann and Sarin, S. Comparative audit: the trouble with POSSUM Journal of Royal Soc of Med 2001: 94(12): 632-634.

2. Yii MK, Ng KJ. Risk-adjusted surgical audit with the POSSUM scoring system in a developing country. Br J Surg 2002; 89: $110-113$.

\begin{tabular}{llllll} 
Mortality & Number & Mean risk (\%) & Predicted & Actual deaths & 0:E ratio \\
\hline group (\%) & of patients & & (expected) deaths & (observed) (\%) & \\
\hline$<10$ & 5 & 7.3 & 0 & $2(40)$ & $5: 48.1$ \\
\hline $10-19$ & 37 & 13.9 & 5 & $10(24)$ & $1.94: 1$ \\
\hline $20-29$ & 14 & 24.5 & 3 & $5(35.7)$ & $1.46: 1$ \\
\hline $30-39$ & 13 & 35.4 & 5 & $9(69.2)$ & $1.96: 1$ \\
\hline $40-49$ & 26 & 44.7 & 12 & $16(61.5)$ & $1.38: 1$ \\
\hline $50-59$ & 16 & 53.6 & 9 & $7(43.8)$ & $0.82: 1$ \\
\hline $60-69$ & 6 & 67.2 & 4 & $4(66.7)$ & $0.99: 1$ \\
\hline $70-79$ & 13 & 74.6 & 10 & $7(53.8)$ & $0.72: 1$ \\
\hline $80-89$ & 18 & 85.9 & 15 & $11(61.1)$ & $0.71: 1$ \\
\hline $90-100$ & 18 & 96 & 17 & $16(88.9)$ & $0.93: 1$ \\
\hline $0-100$ & 166 & 48.3 & 63 & $87(52.4)$ & $1.09: 1$ \\
\hline
\end{tabular}

Table 5: Comparison of observed and predicted morbidity using linear analysis for POSSUM morbidity

\begin{tabular}{llllll} 
Mortality & Number & Mean risk (\%) & Predicted & Actual deaths & 0:E ratio \\
\hline group (\%) & of patients & & (expected) deaths & (observed) (\%) & \\
\hline $10-100$ & 161 & 49.6 & 80 & 85 & $1.06: 1$ \\
\hline $20-100$ & 124 & 60.2 & 75 & 75 & $1: 1$ \\
\hline $30-100$ & 110 & 64.8 & 71 & 73 & $1.02: 1$ \\
\hline $40-100$ & 97 & 68.7 & 67 & 61 & $0.92: 1$ \\
\hline $50-100$ & 71 & 77.5 & 55 & 54 & $1.57: 1$ \\
\hline $0-100$ & 166 & & 347 & 348 & $1: 1$ \\
\hline
\end{tabular}

Table 6: Comparison of observed and predicted morbidity using exponential analysis for POSSUM

O:E Observed: Expected

3. Sagar P, Hartley M, Mancey-Jones B: Comparative audit of colorectal resection with the POSSUM scoring system. Br J Surg 1994;81: 1492-1494.

4. Copeland GP. Comparative audit: fact versus fantasy. $\mathrm{Br} \mathrm{J}$ Surg 1993; 80: 1424-15.

5. Copeland GP, Jones D, Walters M. POSSUM: a scoring system for surgical audit. Br. J. Surg. 1991; 78: 355-60

6. Prytherch D, Whiteley MS, Higgins B, et al. POSSUM and Portsmouth POSSUM for predicting mortality. Physiological and Operative Severity Score for the enUmeration of Mortality and morbidity. Br J Surg 1998; 85: 1217-1220.

7. Whiteley MS, Prytherch D, Higgins B, et al. An evaluation of the POSSUM Surgical Scoring System. Br J Surg 1996: 83; 812-815.

8. Hobson SA, Sutton CD, Garcea G, et al. Prospective comparison of POSSUM and P-POSSUM with clinical assessment of mortality following emergency surgery. Acta Anaesth Scand. 2007: 51: $94-100$ 
Evaluation of POSSUM and P-POSSUM as predictors of mortality and morbidity in patients undergoing laparotomy at a referral hospital in Nairobi, Kenya Kimani $\mathrm{MM}^{1,2}$, Kiiru $\mathrm{JN}^{3}$, Matu $\mathrm{MM}^{3}$, Chokwe $\mathrm{T}^{1,2}$, Saidi $\mathrm{H}^{1,2}$

9. Wijesinghe LD, Mahmood T, Scott DJA, et al. Comparison of POSSUM and the Portsmouth predictor equation for predicting death following vascular surgery. Br J Surg 1998; 83: $209-212$.

10. Hosmer D, Lemeshow S. A goodness of fit test for the multiple logistic regression models. Community Statistics 1980; 10: 1043 - 1069 .

11. Hobson SA, Sutton CD, Garcea G, Thomas WM Prospective comparison of POSSUM and P-POSSUM with clinical assessment of mortality following emergency surgery. Acta Anaesthesiologica Scandinavica. 2007: 51: 94-100

12. Bollschweiler E, Lubke T, Stefan P et al. Holscher Evaluation of POSSUM scoring system in patients with gastric cancer undergoing D2-gastrectomy BMC Surgery 2005, 5:8
13. Vather R, Zargar-Shoshtari K, Adegbola S and Hill AG. Comparison of the POSSUM, P-POSSUM AND CR-POSSUM scoring systems as predictors of postoperative mortality in patients undergoing major colorectal surgery ANZ J. Surg. 2006; 76: 812-816

14. Khan AW, Shah SR, Agarwal AK, et al. Evaluation of the POSSUM Scoring System for Comparative Audit in Pancreatic Surgery Diagnostic Surgery 2003 20:539-545

15. Sagar P, Hartley M, Mancey-Jones B, Comparative audit of colorectal resection with the POSSUM scoring system. Br J Surg 1994;81: 1492-1494.

16. Yeo CJ, Cameron JL, Sohn TA, et al. Six hundred fifty consecutive pancreaticoduodenectomies in the 1990s: Pathology, complications, and outcomes. Ann Surg 1997;226:248-257. 